\title{
Congenital syphilis re-emergence in Winnipeg, Manitoba
}

\author{
Peter Benoit ${ }^{1 *}$, Lana Tennenhouse ${ }^{2}$, Alicia Lapple ${ }^{1}$, Gillian Hill-Carroll1', Souradet Shaw ${ }^{2}$, \\ Jared Bullard ${ }^{2,3}$, Pierre Plourde ${ }^{1,2}$
}

\begin{abstract}
Background: Infectious syphilis rates have been increasing in Winnipeg, Manitoba among individuals during their childbearing years. Untreated or inadequately treated prenatal infection often results in congenital syphilis, with devastating consequences to fetal health and survival. The objective of this study was to review public health surveillance data regarding congenital syphilis incidence and birthing parent risk factors in Winnipeg from 2018 to 2020.

Methods: Data extracted from a population-based surveillance database maintained by the Winnipeg Regional Health Authority Public Health investigations for all 2018-2020 probable or confirmed cases of early congenital syphilis or syphilitic stillbirth were reviewed. Rates of congenital syphilis were calculated per 1,000 live births. Descriptive analyses were performed to describe birthing parent age, neighbourhood of residence, intravenous substance use, Child and Family Services involvement, access to prenatal care and obtainment of adequate prenatal treatment.
\end{abstract}

Results: There were eight cases of confirmed/probable congenital syphilis in 2018, 22 cases in 2019 and 30 cases in 2020. Average birthing parent age was $26.5-27.0$ years. The majority (66.7\%) of birthing parents lived in inner city neighbourhoods with known infectious syphilis outbreaks. Over $50 \%$ of birthing parents did not receive any prenatal care, or the care received consisted of inadequate treatment or follow-up. Reinfection among birthing parents who did receive prenatal care was suspected in an additional $23.3 \%$ of cases.

Conclusion: Congenital syphilis rates in Winnipeg have increased dramatically. Public health and healthcare provider efforts to address the needs of the community are vital for promoting access to safe and effective prenatal care.
This work is licensed under a Creative Commons Attribution 4.0 International License.

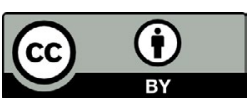

Affiliations

${ }^{1}$ Population and Public Health Program, Winnipeg Regional Health Authority, Winnipeg, MB

2 Max Rady College of Medicine, Rady Faculty of Health Sciences, University of Manitoba, Winnipeg, MB

${ }^{3}$ Cadham Provincial Laboratory, Manitoba Health and Seniors

Care, Winnipeg, MB

\section{*Correspondence:}

pbenoit2@wrha.mb.ca

Suggested citation: Benoit P, Tennenhouse LG, Lapple A, Hill-Carroll G, Shaw SY, Bullard J, Plourde P. Congenital syphilis re-emergence in Winnipeg, Manitoba. Can Commun Dis Rep 2022;48(2/3):89-94.

https://doi.org/10.14745/ccdr.v48i23a06

Keywords: congenital syphilis, surveillance, prenatal care, health inequity

\section{Introduction}

Rates of syphilis have been increasing in Canada; more than doubling between 2016 and 2020 (1), with the most dramatic increases observed in the Prairie Provinces. During the same period, rates of congenital syphilis have increased 10 to 15 -fold, as a consequence of large heterosexual outbreaks of syphilis primarily in the Prairie Provinces.

Congenital syphilis occurs via vertical transmission of the spirochete Treponema pallidum from a pregnant person to a fetus during pregnancy. Manifestations range from asymptomatic infection to spontaneous abortion, stillbirth, and multisystem involvement with severe consequences for infant health (2). Risk of transmission to the fetus is high, with estimates of
$70 \%-100 \%$ among untreated pregnant persons with primary or secondary syphilis, $40 \%$ in early latent syphilis and less than $10 \%$ in late latent syphilis (3). However, congenital syphilis is almost entirely preventable with adequate prenatal treatment, defined as benzathine penicillin $\mathrm{G}$ at least 30 days prior to delivery (4). Screening for infectious syphilis at the first prenatal care appointment is considered standard of care across Canada. Further screening may be considered in high risk/outbreak populations (3). Lack of access to prenatal care has been found to be a large modifiable risk factor for congenital syphilis in the United States (5). Given that the consequences of congenital syphilis are significant, and that effective screening and treatment are available, the occurrence of congenital syphilis cases is an 
important metric to assess the success of our public health and healthcare systems. If functioning optimally, we should see no cases of congenital syphilis.

In Winnipeg, there had been no recorded cases of congenital syphilis since 1977, and more recently the first case detected in Manitoba was in 2015 (in a northern community), with the first case in Winnipeg in 2018. However, there has since been a dramatic increase in infectious syphilis, with an age-standardized rate of 79.5 and 140.4 cases per 100,000 individuals in Winnipeg in 2018 and 2019, respectively (unpublished data, Pierre Plourde). This represents an $81 \%$ increase in cases in 2018 over 2017 and a further almost doubling of cases in 2019. While the majority of cases previously affected men who have sex with men disproportionately, the rate of heterosexual transmission has increased with women comprising $47 \%$ of infectious syphilis cases in Winnipeg in 2018 and 54\% of cases in 2019 (unpublished data, Pierre Plourde), with the first signals of increased transmission in heterosexual women observed as early as 2015 (6). Similar trends of increasing infectious syphilis incidence among women have been noted in other Canadian provinces, including British Columbia and Alberta $(7,8)$. The presence of missed or inadequately treated infectious syphilis among those who are in their childbearing years represents a failure of the healthcare system and signifies the critical importance of intensifying public health and healthcare provider efforts aimed at prevention of congenital syphilis, in collaboration with community-based agencies involved in outreach to pregnant individuals.

Our objective was to review public health surveillance data regarding congenital syphilis incidence in Winnipeg from 2018 to 2020, and to describe the characteristics of pregnant persons giving birth to infants with congenital syphilis. The years 2018-2020 were chosen as a time horizon, as prior to 2018 there were no reported cases of congenital syphilis in Winnipeg for over 40 years.

\section{Methods}

\section{Data source}

Reported cases of congenital syphilis in the surveillance database of the Winnipeg Regional Health Authority were reviewed from January 2018 to December 2020. Winnipeg is the largest city in Manitoba, with a population of about 700,000 in 2016 (9) and 8,675 live births in 2018 and 8,560 live births in 2019, as recorded in the Winnipeg Regional Health Authority Healthy Parenting and Early Child Development program database (personal communication, Dr. Christopher Green, University of Manitoba).

All cases of infectious and congenital syphilis are reportable to Public Health at the provincial level. Cases are classified as Winnipeg cases using postal code of most recent known address, or as individuals known to be living in Winnipeg for the duration of the pregnancy without a Winnipeg address on file. Cases are managed by public health nurses who complete investigation forms, collect sociodemographic characteristics of the individual, and supplement the public health record with relevant clinical notes. For congenital syphilis investigations, public health records are maintained separately for the birthing parent and infant. These records are stored in a population-based surveillance database.

Reported cases of possible congenital syphilis are classified by Manitoba Public Health into confirmed or probable early syphilis, and starting in 2019, also include classifications of confirmed or probable syphilitic stillbirth. Based on the case definitions provided by Manitoba Health and Seniors Care (see Annex)، classification of cases is established by the joint input of a public health nurse and paediatric infectious disease specialist, followed by the review of a medical officer of health.

\section{Measures}

Case investigation public health data reviews for both birthing parent and infant were performed for all confirmed or probable cases of congenital syphilis in Winnipeg between January 2018 and December 2020. At least two individuals reviewed each record independently. Data on the following birthing parent's characteristics were collected: 1) age (years); 2) neighbourhood of residence (Winnipeg Downtown, Point Douglas, or other); 3 ) intravenous substance use over the past year (yes, no); 4) Child and Family Services involvement (yes, no); 5) adequate prenatal treatment defined as receipt of at least one course of benzathine penicillin $\mathrm{G} 2.4$ million units at least 30 days prior to delivery (yes, no, or unknown); and 6) receipt of any prenatal care (none, some but did not receive adequate treatment, some but high risk of re-exposure or reinfection in third trimester not adequately treated, some and treated adequately, or unknown).

\section{Analysis}

Means, medians, interquartile ranges, frequencies and percentages were used to describe the birthing parent characteristics of the study population. The birth rate of infants with congenital syphilis per 1,000 live births was calculated for each year in the surveillance period, using the estimated total number of live births per year in Winnipeg as the denominator (estimated as 8,600 for 2020).

\section{Ethics}

Research ethics approval was not required for analysis and reporting of routinely collected public health surveillance data.

\section{Results}

There were 60 cases of confirmed or probable congenital syphilis in Winnipeg from 2018 to 2020 (Table 1, Figure 1). Eight of these cases occurred in 2018, 22 in 2019 and 30 in 2020. This corresponds to rates of congenital syphilis (per 1,000 live births) of 0.9 in 2018, 2.6 in 2019 and 3.5 in 2020. 
Table 1: Congenital syphilis incident cases and risk factors in Winnipeg, Manitoba from 2018-2020

\begin{tabular}{|c|c|c|c|c|c|c|}
\hline \multirow{2}{*}{ Characteristic } & \multicolumn{2}{|c|}{$\begin{array}{c}2018 \\
(\mathrm{~N}=8) \\
\end{array}$} & \multicolumn{2}{|c|}{$\begin{array}{c}2019 \\
(N=22) \\
\end{array}$} & \multicolumn{2}{|c|}{$\begin{array}{c}2020 \\
(\mathrm{~N}=30)\end{array}$} \\
\hline & $\mathbf{n}$ & $\%$ & $\mathbf{n}$ & $\%$ & $\mathbf{n}$ & $\%$ \\
\hline \multicolumn{7}{|c|}{ Adverse birth outcome } \\
\hline $\begin{array}{l}\text { Confirmed early } \\
\text { congenital syphilis }\end{array}$ & 5 & 62.5 & 13 & 59.1 & 13 & 43.3 \\
\hline $\begin{array}{l}\text { Probable early } \\
\text { congenital syphilis }\end{array}$ & 3 & 37.5 & 5 & 22.7 & 9 & 30.0 \\
\hline $\begin{array}{l}\text { Lab-confirmed } \\
\text { syphilitic stillbirth }\end{array}$ & N/A & N/A & 1 & 4.5 & 6 & 20.0 \\
\hline $\begin{array}{l}\text { Probable syphilitic } \\
\text { stillbirth }\end{array}$ & N/A & $N / A$ & 3 & 13.6 & 2 & 6.7 \\
\hline $\begin{array}{l}\text { Pregnant person's } \\
\text { age (years) } \\
\text { at delivery, } \\
\text { mean/median } \\
\text { (interquartile } \\
\text { range) }\end{array}$ & $26.6 / 27$ & $23-30$ & $27.0 / 26$ & $22-30$ & $26.5 / 25$ & $22-31$ \\
\hline \multicolumn{7}{|c|}{ Neighbourhood of residence } \\
\hline Downtown & 3 & 37.5 & 10 & 45.5 & 10 & 33.3 \\
\hline Point Douglas & 3 & 37.5 & 9 & 40.9 & 5 & 16.7 \\
\hline Other & 2 & 25.0 & 3 & 13.6 & 15 & 50.0 \\
\hline
\end{tabular}

\section{Risk factors}

\begin{tabular}{|l|r|r|r|r|r|r|}
\hline $\begin{array}{l}\text { Intravenous } \\
\text { substance use } \\
\text { over the past year }\end{array}$ & 4 & 50.0 & 9 & 40.9 & 3 & 10.0 \\
\hline $\begin{array}{l}\text { Child and } \\
\begin{array}{l}\text { Family Services } \\
\text { involvement }\end{array}\end{array}$ & 4 & 50.0 & 8 & 36.4 & 4 & 13.3 \\
\hline
\end{tabular}

Adequate treatment 30 days or more prior to delivery

\begin{tabular}{|l|r|r|r|r|r|r|}
\hline Yes & 0 & 0.0 & 6 & 27.3 & 2 & 6.7 \\
\hline No & 8 & 100.0 & 14 & 63.6 & 25 & 83.3 \\
\hline Unknown & 0 & 0.0 & 2 & 9.1 & 3 & 10.0 \\
\hline
\end{tabular}

\begin{tabular}{|l|r|r|r|r|r|r|}
\hline Prenatal care & 3 & 37.5 & 7 & 31.8 & 14 & 46.7 \\
\hline $\begin{array}{l}\text { Some, but did not } \\
\text { receive adequate } \\
\text { treatment }\end{array}$ & 3 & 37.5 & 3 & 13.6 & 3 & 10.0 \\
\hline $\begin{array}{l}\text { Some, but high-risk } \\
\text { re-exposure or } \\
\text { reinfection in } \\
\text { third trimester not } \\
\text { adequately treated }\end{array}$ & 2 & 25.0 & 4 & 18.2 & 8 & 26.7 \\
\hline $\begin{array}{l}\text { Some, treated } \\
\text { adequately }\end{array}$ & 0 & 0.0 & 6 & 27.3 & 2 & 6.7 \\
\hline Unknown & 0 & 0.0 & 2 & 9.1 & 3 & 10.0 \\
\hline
\end{tabular}

Abbreviation: N/A, not available

Median birthing parent age ranged from 26.5 to 27.0 years for each calendar year throughout the surveillance period. The majority of birthing parents lived in two specific neighbourhoods of Winnipeg (Downtown and Point Douglas), both of which are considered to be in the city's inner core and have historically been associated with sexually transmitted infection outbreaks $(5,10)$. These inner-city neighbourhoods are known to have higher rates of poverty, racialized populations and crime. They are also well studied in terms of mortality data and are known to have a significantly lower life expectancy in contrast with the rest of Winnipeg. Intravenous substance use over the
Figure 1: Early congenital syphilis in Winnipeg, Manitoba by month/year of birth, 2018-2020

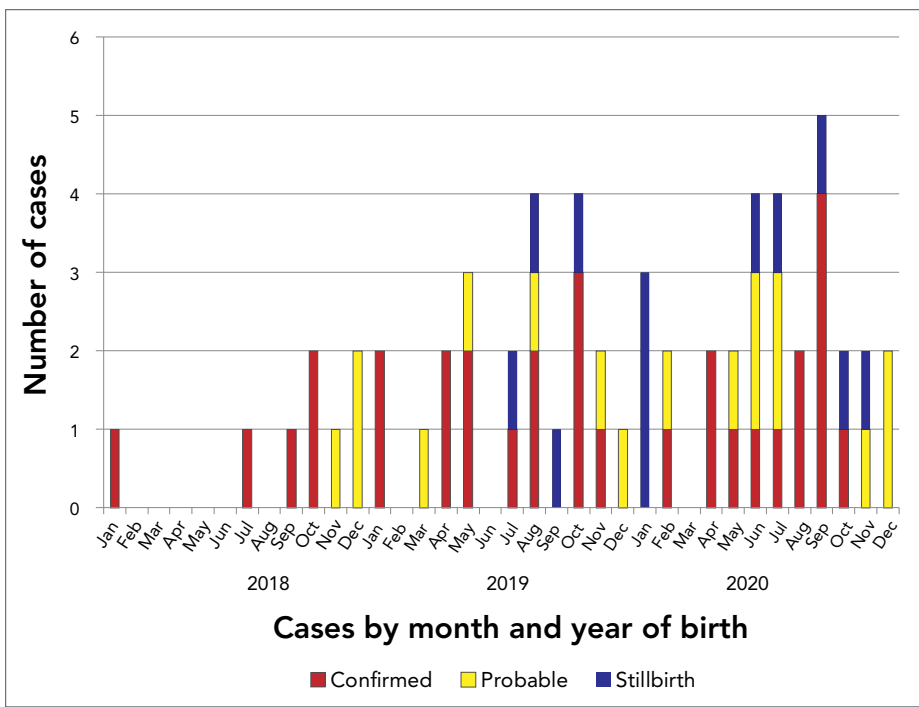

past year was reported in $50.0 \%$ of birthing parents in 2018 , 40.9\% in 2019 and $10.0 \%$ in 2020. Child and Family Services were involved with $50.0 \%$ of cases in $2018,36.4 \%$ in 2019 and $13.3 \%$ in 2020. Of the 16 birthing parents who reported intravenous substance use, 11 (69\%) named crystal methamphetamine.

Over $50 \%$ of birthing parents did not receive any prenatal care or received only limited prenatal care (usually one visit) with inadequate follow-up and treatment. On average from 2018 to $2020,23.3 \%$ of birthing parents received prenatal care; however, a reinfection or high-risk re-exposure was suspected to have occurred in the third trimester. None of the birthing parents received adequate treatment during pregnancy in 2018 , although this increased to $27.3 \%$ in 2019 , then declined again to $6.7 \%$ in 2020 .

\section{Discussion}

In 2007, the World Health Organization published the goal of decreasing congenital syphilis rates to 0.5 cases or less per 1,000 live births (11). With increasing cases of congenital syphilis each year and a rate of 3.5 cases per 1,000 live births in 2020, Winnipeg is far from meeting this goal. Young birthing age, residence in select inner city neighbourhoods with large infectious syphilis outbreaks, intravenous substance use and involvement with Child and Family Services emerged as possible birthing parent co-factors in this population. This strongly suggests that poverty and social structural disadvantages play a significant role in the occurrence of congenital syphilis.

Lack of access to adequate prenatal care during pregnancy was present in over $50 \%$ of those assessed during the surveillance period. Inequities in prenatal care access are a known issue in Winnipeg, with research indicating that $15 \%-21 \%$ of pregnant persons residing in Winnipeg's inner core received inadequate 
prenatal care from 2004-2005 to 2008-2009 (12). Those who are most vulnerable may have trouble accessing the healthcare system for a variety of reasons, including lack of transportation, language difficulties, concern for healthcare provider prejudices arising from racism and lack of knowledge about the healthcare system (13). Therefore, effective approaches to the management of rising rates of congenital syphilis must take into account the unique needs of the community. In Alberta, a program was implemented that provided prenatal care to street-involved individuals with the goal of determining the feasibility of decolonizing prenatal care by performing outreach through inner city community members (14). Study participants reported high satisfaction rates with the program, and particularly emphasized their appreciation that care providers were supportive and nonjudgmental. In Winnipeg, collaborative efforts between public health and Indigenous community organizations are underway with the goal of establishing Indigenous-led approaches to sexually transmitted and bloodborne infection management; however, challenges in securing funding for Indigenous-led sexually transmitted and bloodborne infection strategies continue to be a significant challenge.

A subset of the population who did receive prenatal care were suspected to have been re-exposed to infectious syphilis sometime after their first negative prenatal screens. Several individuals were treated with documented reduction in serologic response during pregnancy, followed by serologic evidence of reinfection (i.e. rising titres after titres had decreased). It is also possible that some individuals with negative syphilis serology at first prenatal visit had already developed infectious syphilis at the time of initial screening, but were too early in their infection to have developed a detectable immune response. A report from New York City indicated that about one-third of their cases of congenital syphilis occurred during pregnancies that initially screened negative (15). These results highlight the importance of repeat infectious syphilis screening during pregnancy among those living in communities with known outbreaks and other risk factors. In light of increasing congenital syphilis rates, since early 2019, healthcare providers in Winnipeg have been advised to test all pregnant individuals for infectious syphilis at 28 weeks gestation and at delivery in addition to routine first prenatal visit screening.

Data on ethnicity was not collected consistently during the surveillance period. This represents an important missed opportunity. Racialized groups have been found to disproportionately bear the burden of congenital syphilis, with a report indicating that $85 \%$ of those who gave births to infants with congenital syphilis in Los Angeles were either Latin American or African American (16). In Canada, it is known that Indigenous individuals have barriers to accessing safe healthcare due to experiences of racism in a colonialist system (17). This highlights the importance of systematically collecting data on ethnicity in future surveillance of congenital syphilis to help ensure that subsequent interventions and care models are provided in culturally safe and appropriate manners. Collaboration with Indigenous and other racialized groups would be vital for ensuring this data is collected and reported in a respectful and non-stigmatizing way.

\section{Strengths and limitations}

This surveillance data had strengths and limitations. Data on ethnicity was not collected consistently. Data on intravenous substance use was collected through surveillance data chart review and was reliant on clients self-reporting this activity. As a result, it is possible that intravenous substance use was under-reported. Some potential outcomes of congenital syphilis such as spontaneous abortion were not captured. As well, syphilitic stillbirths were not included in the surveillance database in 2018 and possibly in early 2019, which could have resulted in an underreporting of congenital syphilis. Finally, changes in public health case investigation forms and methods of data entry over the surveillance period may have led to possible inconsistencies in birthing parent characteristic data reporting between the years. A strength of the study includes the use of population-based data with rigorously defined classifications of congenital syphilis cases. Also, the current co-director of the provincial public health laboratory, where all congenital syphilis cases are tested, is a paediatric infectious disease specialist, which should ensure maximal accuracy in the classification of cases.

\section{Conclusion}

Congenital syphilis rates are increasing in Winnipeg, alongside the increase in infectious syphilis rates among individuals during their childbearing years. Lack of access to prenatal care emerged as a major risk factor for congenital syphilis. The importance of repeat screening for infectious syphilis during pregnancy in highrisk populations was also demonstrated. Addressing systemic barriers to prenatal care and implementing appropriate culturally safe care models informed by the needs of the community may be effective interventions to contend with increasing congenital syphilis rates. Future studies and interventions should emphasize a community-based research approach, where early community involvement is a key priority to connect with individuals at highest risk. This will allow researchers to learn more about key factors that increase the risk of congenital syphilis and provide access to resources in a way that is most accessible for those who traditionally do not access key resources such as prenatal care. Without these changes in approach, traditional colonialist interventions may not be effective to address rising congenital syphilis rates.

\section{Authors' statement}

PB - Analyzed and interpreted the surveillance data, drafted first copy of the manuscript, data analysis and manuscript revisions

LGT - Analyzed and interpreted the surveillance data, drafted first copy of the manuscript, data analysis and manuscript revisions 
$\mathrm{AL}$ - Investigation, provided clinical expertise, and provided manuscript revisions

GHC - Investigation, provided clinical expertise, and provided manuscript revisions

JB - Investigation, provided clinical expertise, and provided manuscript revisions

SYS - Provided academic and methodologic expertise, data analysis, and manuscript revisions

PP - Conceptualized the project, supervised

All authors contributed equally to this surveillance analysis.

The content and view expressed in this article are those of the authors and do not necessarily reflect those of the Government of Canada.

\section{Competing interests}

None.

\section{Acknowledgements}

We would like to thank the Healthy Sexuality and Harm Reduction team of public health nurses at the Winnipeg Regional Health Authority (WRHA) who worked diligently to gather the surveillance data used in this report. For persons of reproductive age who are unable to access appropriate prenatal care we acknowledge the significant impact this has on their health and the health of their infants. We believe that key barriers can and should be addressed and that this should be a key priority for public health and healthcare systems in Manitoba and Canada.

\section{Funding}

SYS is supported by a Canada Research Chair in Program Science and Global Public Health (Tier II).

\section{References}

1. Public Health Agency of Canada. Infectious Syphilis and Congenital Syphilis in Canada, 2020 (infographic). Ottawa (ON): PHAC; 2021. https://www.canada.ca/en/public-health/services/ publications/diseases-conditions/infectious-syphilis-congenitalsyphilis-canada-2020.html

2. Arnold SR, Ford-Jones EL. Congenital syphilis: A guide to diagnosis and management. Paediatr Child Health 2000;5(8):463-9. DOl PubMed

3. Robinson JL; Canadian Paediactric Society, Infectious Diseases and Immunization Committee. Congenital syphilis: no longer just of historical interest. J Paediatr Child Health 2009;14(5):337. https://cps.ca/en/documents/position/congenital-syphilis

4. Wendel GD Jr, Sheffield JS, Hollier LM, Hill JB, Ramsey PS, Sánchez PJ. Treatment of syphilis in pregnancy and prevention of congenital syphilis. Clin Infect Dis 2002;35 Suppl 2:S200-9. DOI PubMed
5. Kimball A, Torrone E, Miele K, Bachmann L, Thorpe P, Weinstock H, Bowen V. Missed Opportunities for Prevention of Congenital Syphilis - United States, 2018. MMWR Morb Mortal Wkly Rep 2020;69(22):661-5. DOI PubMed

6. Shaw SY, Ross C, Nowicki DL, Marshall S, Stephen S, Davies C, Riddell J, Bailey K, Elliott LJ, Reimer JN, Plourde PJ. Infectious syphilis in women: what's old is new again? Int J STD AIDS 2017;28(1):77-87. DOI PubMed

7. Grennan T, Giesbrecht E, Ogilvie G, Pederson A, van Schalkwyk J, Gilbert M, Wong J. Syphilis outbreak in $\mathrm{BC}$ : Changes to syphilis screening in pregnancy. BCMJ 2019;61(8):328. https://bcmj.org/bccdc/syphilis-outbreak-bcchanges-syphilis-screening-pregnancy

8. Singh A, Romanowski B. The return of syphilis in Canada: A failed plan to eliminate this infection. JAMMI 2019;4(4):215-7. $\mathrm{DOI}$

9. Statistics Canada. Census Profile. 2016 Census. Winnipeg, CY [Census subdivision], Manitoba and Canada [Country] (table). Statistics Canada Catalogue no. 98-316-X2016001. Ottawa (ON): StatCan; 2017. https://www12.statcan.gc.ca/censusrecensement/2016/dp-pd/prof/index.cfm?Lang $=\mathrm{E}$

10. Elliott LJ, Blanchard JF, Beaudoin CM, Green CG, Nowicki DL, Matusko P, Moses S. Geographical variations in the epidemiology of bacterial sexually transmitted infections in Manitoba, Canada. Sex Transm Infect 2002;78 Suppl 1:i139-44. DOI PubMed

11. World Health Organization. The global elimination of congenital syphilis: rationale and strategy for action. Geneva $(\mathrm{CH})$ : WHO; 2007. https://www.who.int/reproductivehealth/publications/ rtis/9789241595858/en/

12. Heaman MI, Martens PJ, Brownell MD, Chartier MJ, Thiessen KR, Derksen SA, Helewa ME. Inequities in utilization of prenatal care: a population-based study in the Canadian province of Manitoba. BMC Pregnancy Childbirth 2018;18(1):430. DOI PubMed

13. Loignon C, Hudon C, Goulet É, Boyer S, De Laat M, Fournier $\mathrm{N}$, Grabovschi C, Bush P. Perceived barriers to healthcare for persons living in poverty in Quebec, Canada: the EQUlhealThY project. Int J Equity Health 2015;14(1):4. DOI PubMed

14. Mill J, Singh A, Taylor M. Women in the Shadows. Can J Urban Res 2012;21(2):68-89. https://www.jstor.org/stable/26193913

15. Slutsker JS, Hennessy RR, Schillinger JA. Factors Contributing to Congenital Syphilis Cases - New York City, 2010-2016. MMWR Morb Mortal Wkly Rep 2018;67(39):1088-93. DOI PubMed

16. Los Angeles County Department of Public Health Division of HIV and STD Programs. Eliminating congenital syphilis in Los Angeles County: A call to action. Los Angeles (CA): LA County Public Health; 2020. http://publichealth.lacounty.gov/dhsp/ Providers/CS_EliminationPlanDraft_01282020.pdf

17. Allen L, Hatala A, ljaz S, Courchene ED, Bushie EB. Indigenous-led health care partnerships in Canada. CMAJ 2020;192(9):E208-16. DOl PubMed 


\section{Annex: Manitoba Health and Seniors Care congenital syphilis case definitions}

\section{Laboratory-confirmed case-early congenital syphilis (within two years of birth)}

- Identification of T. pallidum by dark-field microscopy, direct fluorescence antibody, or detection of T. pallidum DNA by nucleic acid amplification test (e.g. polymerase chain reaction; PCR) in an appropriate clinical specimen, or equivalent examination of material from nasal discharges, skin lesions, placenta or umbilical cord, or autopsy material of a neonate (up to four weeks of age)

OR

- Reactive serology (treponemal and nontreponemal) from venous blood (not cord blood) in an infant/child with or without clinical, other laboratory, or radiographic evidence consistent with congenital syphilis* but who has one or both of the following:

- Rising syphilis serologic titres upon follow-up where there is evidence that the mother had a syphilis infection during pregnancy

- Titres greater than or equal to fourfold higher than those of the mother when collected at the same time or within a week, in the immediate post-natal period

OR

- Reactive serology (treponemal and nontreponemal) from venous blood (not cord blood) in an infant/child with clinical, other laboratory, or radiographic evidence consistent with congenital syphilis* whose mother was seropositive or PCR OR positive for syphilis during pregnancy or at delivery

- A child who does not meet the above criteria but has persistently reactive treponemal serology between 18 and 24 months of age (regardless of maternal treatment status and infectious status)

* Includes any evidence of congenital syphilis on physical examination (e.g. hepatosplenomegaly, consistent rash, condyloma lata, snuffles, pseudoparalysis), evidence of congenital syphilis on radiographs of long bones, a reactive cerebrospinal fluid (CSF) Venereal Disease Research laboratory test (VDRL), an elevated CSF cell count or protein without other cause.

\section{Probable case-early congenital syphilis (within two years of birth) ${ }^{\dagger}$}

- Reactive serology (treponemal and nontreponemal) from venous blood (not cord blood) in an infant/child without clinical, laboratory, or radiographic manifestations of congenital syphilis whose mother had:

OR

○ Untreated or inadequately ${ }^{\ddagger}$ treated syphilis at delivery

- Evidence of reinfection or relapse in the pregnancy following appropriate treatment (e.g. rising nontreponemal titres at least four-fold higher)

+ A persistent treponemal serologic reaction at 18-24 months of age confirms the diagnosis of congenital syphilis. An absent serologic reaction (both treponemal and nontreponemal tests) at, or before, 18-24 months of age excludes the case (i.e. it is no longer probable case).

₹Inadequate treatment consists of any non-penicillin therapy or penicillin administered during pregnancy but less than 30 days before delivery, or despite treatment there has been an inadequate drop in nontreponemal titres. Note: the type of penicillin administered is important; it is usually benzathine penicillin in pregnancy, with the exception of treatment for neurosyphilis.

\section{Laboratory-confirmed case-syphilitic stillbirth}

- A fetal death that occurs after 20 weeks gestation with laboratory confirmation of infection (i.e. detection of T. pallidum DNA in an appropriate clinical specimen, direct fluorescent antibody or equivalent examination of material from placenta, umbilical cord or autopsy material).

\section{Probable case-syphilitic stillbirth}

- A fetal death that occurs after 20 weeks gestation where the mother had untreated or inadequately treated syphilis prior to delivery OR whose mother had evidence of reinfection or relapse in pregnancy following appropriate treatment (such as rising titres), with no other cause of stillbirth established

Source: https://www.gov.mb.ca/health/publichealth/cdc/ protocol/syphilis.pdf 